\title{
GESTÃO DO CONHECIMENTO E PROCESSOS UNIVERSITÁRIOS
}

DOI: http://dx.doi.org/10.18616/pers10

Michele Schneider - Unesc

E-mail: micheleschneier@unesc.net

Júlio Cesar Zilli - Unesc

E-mail: zilli42@hotmail.com

Adriana Carvalho Pinto Vieira - Unesc

E-mail: dricpvieira@unesc.net 


\section{INTRODUÇÃO}

A Gestão do Conhecimento (GC), para Nonaka e Takeuchi (1997), trata-se de um processo que permite a identificação, criação, compartilhamento e aplicação dos conhecimentos nos níveis individual, grupal e organizacional. Essa temática assume relevância no meio empresarial quando as organizações conseguem atrelar os impactos da GC com a geração de valor para a organização, de forma a modificar as estratégias organizacionais e proporcionar um diferencial competitivo (COSTA et al., 2009).

A Universidade por sua vez, tem na sua essência a geração e o compartilhamento do conhecimento, onde é esse o ambiente propício para que os conhecimentos que são gerados sejam compartilhados por meio do ensino, pesquisa e extensão (HARDY; FACHIN, 2000). Embora esse seja um dos papéis da academia, nem sempre a GC é tratada com a devida atenção no âmbito da gestão, visto que em muitos casos a gestão acadêmica é desempenhada por professores. Hardy e Fachin (2000) ainda destacam a complexidade da gestão universitária em comparação às organizações convencionais, devido à quantidade de setores e pessoas com diversidade de interesses, objetivos e especificidades técnicas de cada curso, além da sobrecarga de atividades técnicas, participação em conselhos, projetos e o exercício da docência, os quais, por vezes sobrecarregam os gestores, não sendo priorizados os processos que envolvem a GC.

Além da complexidade da gestão acadêmica, pode-se somar aos desafios para a Gestão do Conhecimento, que, de acordo com Souza (2006), estão relacionados em três problemáticas centrais: as bases da Gestão do Conhecimento, os processos de mensuração de resultados e a necessidade de compartilhamento de conhecimento.

Para tanto, buscou-se neste artigo um modelo para diagnosticar a Gestão do Conhecimento presente na gestão acadêmica dos cursos de Tecnologia em Gestão de uma Universidade do sul catarinense. O modelo escolhido para esta análise foi o Diagnóstico de Gestão do Conhecimento de Bukowitz e Williams (2002). Por meio desse modelo, será averiguada como está instituída a Gestão do Conhecimento na gestão acadêmica. 


\section{REVISÃO TEÓRICA}

\section{Conceito DE UNIVERSIDADE}

O ensino superior evoluiu ao longo dos tempos passando por diversos modelos, iniciando com o pioneirismo da Escola Escolástica, oriunda da criação das Universidades Bolonha e Paris, no Século XI; o Século XVIII é marcado pelo lluminismo e a academia passa por transformações, chegando ao modelo de Universidade Arte-Cultura. A Revolução Industrial no Século XIX impulsiona o surgimento da Universidade Científico-Tecnológica, cujo objetivo é o treinamento de profissionais técnico administrativos, modelo consolidado com a segunda Guerra Mundial. Esse modelo entra em falência com a Crise Estudantil de 1968, momento em que surge a Universidade Empreendimento. Nesse estágio, a Universidade muda seu conceito para abarcar a maior quantidade de alunos, permitindo o acesso àqueles que antes não eram atendidos pelo ensino superior (ALMEIDA, 2014).

Para fins de conceito, será tomado como base as definições legais presentes no Brasil, a Lei de Diretrizes e Bases da Educação (LDB) (BRASIL, 1996, n. p on-line) define Universidade como sendo (art. 52): "[...] uma instituição pluridisciplinar de formação dos quadros de profissionais de nível superior, de pesquisa, de extensão e de domínio e cultivo do saber humano." Outra definição legal vigente pode ser encontrada no decreto n. 5.773/06, em que as universidades são caracterizadas pela indissociabilidade do ensino, pesquisa e extensão, atuando de forma pluridisciplinar na formação de profissionais de nível de ensino, pesquisa e extensão.

A Universidade como organização é composta pela gestão acadêmica, que compreende o planejamento e a execução das atividades técnico-administrativas e a docência, que compreende o fazer pedagógico.

\section{CONCEITOS DE GESTÃO UNIVERSITÁRIA}

A gestão universitária, geralmente, é desempenhada por docentes e, estes são responsáveis por atender as demandas em condições favoráveis ou não, visando alcançar os objetivos instituições, por meio de práticas, ações gerenciais e decisões, que muitas vezes acabam sendo tomadas com base no empirismo (MARRA; MELO, 2005).

Para Miranda (2010), a gestão universitária é assumida por professores os quais devem possuir habilidades técnicas como docentes, pesquisadores e 
gestores. A problemática que envolve a gestão acadêmica nos moldes citados por Miranda (2010), geralmente está relacionada à falta de treinamento para a execução das atividades técnico-administrativas e gerenciais, e boa parte do processo de aprendizagem se dá por meio da prática em tentativas de erro e acerto. No entendimento de Tardif (2000), o autor destaca que o trabalho em sala de aula exige do docente uma série de habilidade e competências para o convívio em sala de aula, iteração com os alunos, normas e regras sociais, que podem auxiliar e facilitar o trabalho dos professores na gestão universitária.

Relata em seus estudos Jacometti (2012) que as estruturas organizacionais acadêmicas são constantemente ajustadas de acordo com as demandas do mercado e sociedade, bem como ao alinhamento estratégico institucional ao qual estão inseridos. Nesse sentido, à medida que as demandas são alteradas, a academia precisa se adequar às novas exigências. No entanto, vale ressaltar que o ritmo das mudanças nos dias atuais tem se tornado cada vez mais intenso.

Ressalta-se que a formulação das estratégias nas organizações acadêmicas difere de organizações industriais e comerciais, pois existe nesse contexto uma série de variações, tendo em vista a quantidade de membros envolvidos e as estratégias, costumeiramente, são fragmentadas em departamentos, normas reguladoras profissionais, as quais influenciam fortemente na determinação das estratégias, dificultando as mudanças estratégicas (HARDY; FACHIN, 2000).

As Instituições de Ensino Superior apresentam algumas questões em termos de gestão, semelhantes as organizações industriais e comerciais relacionadas aos processos estratégicos e táticos, e da mesma forma que as empresas citadas anteriormente preocupam-se com as mudanças, desafios e tendências na gestão, dessa forma é perceptível a preocupação com o tema Gestão do Conhecimento (BRITO; BOLSON, 2014).

Destacam Melo, Lopes e Ribeiro (2013) que o trabalho dos gestores universitários está atrelado ao desejo de cumprir as metas e demandas da gestão, aliados às restrições orçamentárias, de pessoal e políticas institucionais, as quais acabam limitando a atuação do gestor. Os desafios da gestão de acordo com Saussois (1998) passam por três formas de desafios, os quais derivam das dificuldades de coordenação das atividades, da busca pela cooperação dos membros da equipe em prol do atendimento das metas e dos objetivos da organização e/ou departamento, bem como das ações para promover a cultura da organização no contexto do trabalho de cada indivíduo. 
Dessa forma, de acordo com os autores supracitados, no exercício da gestão acadêmica é comum os gestores assoberbarem-se de trabalhos técnicos administrativos e descuidarem-se da Gestão do Conhecimento.

\section{GESTÃO DO CONHECIMENTO}

A Gestão do Conhecimento é definida por Terra (2000) como um processo de identificação, codificação, maximização e compartilhamento do conhecimento organizacional. A temática vem ganhando destaque devido ao uso maciço da tecnologia de informação, que segundo Dihl, Holanda e Francisco (2010), uma mudança de paradigmas da era industrial para a era do conhecimento, no qual as organizações estão passando na atualidade, com destaque na criação e disseminação do conhecimento.

Brito e Bolson (2014) transcorrem a respeito da gestão do conhecimento como sendo algo imprescindível para o sucesso das organizações, haja vista os processos que são necessários desenvolver para a efetividade da gestão do conhecimento. As empresas cada vez mais estão compreendendo a importância em aproveitar e utilizar todo conhecimento gerado na organização, bem como o conhecimento que os indivíduos trazem consigo. Esse conhecimento de acordo com Terra (2000) compreende os níveis individual, grupal e organizacional. Segundo o autor, o conhecimento individual é aquele que o indivíduo desenvolve por meios formais de educação (graduação, especialização, cursos, treinamento, entre outros) e pela experiência adquirida ao longo de sua trajetória profissional e pessoal. Ao ser inserido em um grupo o indivíduo transfere seus conhecimentos e recebe os conhecimentos do grupo, desde que haja condições para que isso ocorra, esse processo de trocas de experiências e conhecimentos caracteriza, de maneira geral, o compartilhamento do conhecimento. E ainda há os conhecimentos gerados na organização e para a organização, os quais são frutos da mescla dos conhecimentos individuais e grupais (TERRA, 2000).

Os conhecimentos gerados em uma organização, quando sistematizados, reduzem as incertezas e otimizam o processo de tomada de decisão, dessa forma a Gestão do Conhecimento pode representar um diferencial competitivo, pois o sucesso institucional está intimamente ligado com a capacidade de aproveitar-se das oportunidade e potencialidades do mercado (SILVEIRA; NETO, 2013).

Os desafios da Gestão do Conhecimento, portanto, passam por uma série de questões estruturais, nos quais as organizações precisam se preocupar, com a 
necessidade de que as lideranças sejam observadas e trabalhadas, no estimulo e disseminação da cultura voltada para o conhecimento e o compartilhamento do conhecimento (SOUZA, 2006).

\section{DIAGNÓSTICO DA GESTÃO DO CONHECIMENTO (DGC)}

O modelo de Diagnóstico de Gestão do Conhecimento (DGC) de Bukowitz e Williams (2002) identifica a gestão do conhecimento como um processo de geração de riqueza em uma organização, a partir do conhecimento ou capital intelectual, e baseia-se no construto da compreensão diária de que novas experiências geradas nas organizações são capazes de produzir novos conhecimentos; com base na capacidade de aprender com as experiências e novos conhecimentos, reter, adaptar-se e descartar os conhecimentos desnecessários.

Para as autoras, o Diagnóstico da Gestão do Conhecimento (DGC) tem por objetivo estruturar os processos da gestão do conhecimento e é baseado em sete seções, distribuídos nos processos táticos (obtenha, utilize, aprenda e contribua), e estratégicos (avalie, construa/mantenha e descarte). Os processos táticos são diretamente ligados às atividades do cotidiano, de forma que o indivíduo reúna as informações necessárias para realizar suas atividades, gerar valor e desenvolver os conhecimentos necessários. Os processos estratégicos exigem uma avaliação contínua do capital intelectual existente e uma comparação com necessidades futuras (BUKOWITZ; WILLIAMS, 2002).

Seção 1 - Obtenha: essa seção visa identificar como a organização lida com o grande volume de informações disponíveis e seleciona as que são fundamentais para o desenvolvimento das atividades no momento em que forem necessárias. O grande desafio das organizações apontado por Bukowitz e Williams (2002) está relacionado à maneira como a organização encontra, gerencia e disponibiliza as informações contidas nos sistemas.

Seção 2 - Utilize: a seção apresenta como premissa identificar como a organização se utiliza de mecanismos inovadores para gerar valor aos negócios e de que forma são disponibilizados e criados espaços que permitam a geração e utilização do conhecimento.

Seção 3 - Aprenda: a seção envolve identificar e compreender os processos e estilos de aprendizagem da organização em prol da Gestão do Conhecimento. 
Seção 4 - Contribua: a problemática envolvida nessa seção está em que a organização e seus colaboradores devem compartilhar o conhecimento para que sejam gerados novos conhecimentos.

Seção 5 - Avalie: como a organização e os indivíduos fazem para medir o capital intelectual, buscando de forma menos complexa indicadores de medição para que os indicadores sirvam de parâmetro para planejamentos futuros.

Seção 6 - Construa/Mantenha: a problemática envolvida nessa seção está em quantificar e mensurar quanto de investimento é necessário para a manutenção e o crescimento dos ativos intangíveis da organização. Nesse quesito, cabe às pessoas desenvolverem novos experimentos para a geração de valor organizacional e à organização estruturar planos de ação para a manutenção e geração de novos conhecimentos.

Seção 7 - Descarte: a seção visa avaliar como a organização e as pessoas descartam os conhecimentos desnecessários, sendo que a problemática está diretamente relacionada em identificar quais conhecimentos são desnecessários e podem ser descartados.

O Diagnóstico da Gestão do Conhecimento (DGC) permite identificar em qual fase a organização está mais focada e, da mesma forma, qual a fase apresenta maiores fragilidades na Gestão do Conhecimento e que, por consequência, merecem especial atenção.

\section{PROCEDIMENTOS METODOLÓGICOS}

A metodologia aplicada para a obtenção dos objetivos propostos para a pesquisa é definida quanto aos fins, quanto aos procedimentos e quanto à abordagem como Descritiva, Estudo de caso, de campo e qualitativa. A pesquisa descritiva, de acordo com Fachin (2003), tem como finalidade apresentar a população a ser pesquisada, por meio do uso de coleta de dados e, também, aplicação do questionário, contendo 140 questões; divididas em 7 seções, de acordo com o modelo, com o intuito de atender aos objetivos propostos pelo estudo.

Gil (2006) define a pesquisa de estudo de caso como uma investigação de processos na sua totalidade ou em partes do processo, com a finalidade de estudar os fenômenos e fatos sociais envolvidos.

Com o intuito de atender aos objetivos da pesquisa, foi aplicado o questionário que compõe o Diagnóstico de Gestão do Conhecimento (DGC) aos dois gestores dos 
cursos de Tecnologia em Gestão de uma universidade do sul do estado de Santa Catarina e há dois funcionários técnicos administrativos, esse universo pesquisado representa o censo. Os dados foram tabulados e apresentados na sequência.

De acordo com o modelo de Diagnóstico de Gestão do Conhecimento (DGC) de Bukowitz e Williams (2002), cada questão apresenta como opção de resposta as alternativas FS (quando o quesito é fortemente encontrado na organização), $M$ (moderadamente) e Fr (fracamente). Após a aplicação do questionário, os dados são tabulados, levando em consideração as respostas dos respondentes. Aplica-se um peso para cada resposta, em que FS equivale a 3 pontos, M equivale a 2 pontos e Fr equivalente a 1 ponto. De acordo com a quantidade de respondentes, essa pesquisa delineou como parâmetro de pontuação máxima 240 pontos por seção (20 questões $\times 3$ pontuações máximas $\times 4$ respondentes) e 1.680 no geral (240 pontuações individual máxima $x 7$ seções). Com base nos resultados, foi possível chegar aos percentuais por seção.

A instituição de Ensino Superior está localizada na região sul de Santa Catarina há 45 anos, e há 10 tem no seu rol de cursos a categoria de Cursos de Tecnologia em Gestão. Ao longo da história dos cursos de tecnologia, sete coordenadores já atuaram na função. Atualmente, são dois coordenadores para cinco cursos de Tecnologia em Gestão e dois funcionários técnico-administrativos, que realizam as funções de secretaria dos cursos. Um coordenador exerce a coordenação de dois cursos e a coordenação adjunta de outros dois cursos; e o segundo coordenador exerce a coordenação de três cursos. Destes, em dois há assessoria do outro coordenador; nesse contexto, grande parte das decisões estratégicas é tomada em conjunto.

\section{RESULTADOS E DISCUSSÃO}

Após a aplicação dos questionários, foi possível a tabulação e análise dos dados, seguindo as considerações do modelo de Diagnóstico de Gestão do Conhecimento (DGC) de Bukowitz e Williams (2002), os quais são apresentados a seguir:

De acordo com os dados da seção Obter, é possível perceber que a gestão do curso consegue trabalhar relativamente bem a Gestão do Conhecimento, visto que a média da seção aponta para $67,08 \%$. No entendimento de Bukowitz e Williams (2002), um desempenho com média aceitável oscila entre 30 e $70 \%$. Vale destacar alguns itens críticos, os quais tiveram desempenho com média menor na seção. Dentre eles a falta de distinção entre qual informação deve ser controlada 
centralmente e quais informações devem ser livres para serem documentadas e compartilhadas. É possível identificar também falhas da documentação da expertise dos indivíduos, assim como falha na falta de regras para a documentação e compartilhamento do conhecimento.

Tabela 1 - Resumo da pontuação da seção Obter

\begin{tabular}{l|c|c|c}
\hline \multicolumn{1}{c}{ SEÇÃO: OBTER } \\
\hline NÚMERO DE OCORRÊNCIAS & RESULTADO & PESO & TOTAL \\
\hline Morte & 20 & 3 & 60 \\
\hline Fraco & 41 & 2 & 82 \\
\hline Total & 19 & 1 & 19 \\
\hline Pontuação Máxima & & & 161 \\
\hline \% da Secção & & 240 \\
\hline
\end{tabular}

Fonte: Elaborado pelos autores a partir da pesquisa.

A maior incidência de respostas nas questões relativas à seção Obter foram na alternativa Moderado, ocorrendo 41 vezes, obtendo 82 pontos. Nesse ponto, é possível perceber que não existem questões que comprometam fortemente o processo de Gestão do Conhecimento. Porém, é importante que os gestores do curso revejam cada ponto citado como fraco e moderado para definir claramente os objetivos da Gestão do Conhecimento.

A seção Utilizar apresenta desempenho médio de 71,67\%, desempenho este maior que a seção anterior, com destaque para o entendimento dos indivíduos de que as mudanças no espaço de trabalho ocorrem em razão da necessidade de trabalho em equipe e corte de gastos (91,67\%). E merecem atenção especial, por apresentarem baixo desempenho, as questões relativas aos status que os escritórios representam, e esse fator é representativo para a Universidade, pois os cargos e espaços representam status na hierarquia. Existe discordância entre os respondentes quanto ao engessamento e estruturação das reuniões, que podem em alguns casos não favorecer um ambiente criativo e que permita a resolução de problemas; o mesmo ocorre com o envolvimento dos clientes (acadêmicos) na gestão dos cursos e no desenvolvimento de novos produtos (novos cursos de extensão e graduação tecnológica). Outro ponto que merece atenção está na percepção dos respondentes quanto à lucidez na tomada de decisão e resolução dos problemas. 
Tabela 2 - Resumo da pontuação da seção Utilizar

\begin{tabular}{l|c|c|c}
\hline \multicolumn{1}{c}{ SEÇÃO: UTILIZAR } \\
\hline NÚMERO DE OCORRÊNCIAS & RESULTADO & PESO & TOTAL \\
\hline Forte & 27 & 3 & 81 \\
\hline Moderado & 38 & 2 & 76 \\
\hline Fraco & 15 & 1 & 15 \\
\hline Total & & & 172 \\
\hline Pontuação Máxima & & 240 \\
\hline \% da Seç̧ão & & 71,67 \\
\hline
\end{tabular}

Fonte: Elaborado pelos autores a partir da pesquisa.

A seção Utilizar apresentou desempenho médio de 71,67\%, obtendo 172 pontos dos 240 possíveis. Novamente, como na seção Obter, a questão com maior incidência está na reposta Moderado, o que é possível deduzir que a gestão consegue utilizar os conhecimentos produzidos e gerados. Porém, há um longo caminho a ser percorrido para a instituição de processos, claramente percebidos pelos indivíduos, no sentido de canalizar os esforços e estabelecer uma gestão efetiva do conhecimento organizacional.

A seção Aprender tem o objetivo de identificar como a organização desenvolve a aprendizagem organizacional e quais os estilos de aprendizagem utilizados para o favorecimento da gestão do conhecimento (BUKOWITZ; WILLIAMS, 2002). Na gestão dos cursos Tecnológicos, alguns pontos referentes à aprendizagem organizacional merecem atenção especial, com destaque para o processo de aprendizado com os erros e acertos nos projetos e ações desenvolvidas. Os respondentes julgam ser fundamental para o estabelecimento da memória organizacional e, consequentemente, para a Gestão do Conhecimento, que os resultados das ações e projetos sejam registrados, bem como discutidos, pois dessa forma ações que dão resultados favoráveis podem ser reproduzidas; as ações que não surtiram efeitos, e, nem resultados satisfatórios, devem ser evitadas e os erros devem servir como aprendizado. 
Tabela 3 - Resumo da pontuação da seção Aprender

\begin{tabular}{l|c|c|c}
\hline \multicolumn{1}{c}{ SEÇÃO: APRENDER } \\
\hline FómERO DE OCORRÊNCIAS & RESULTADO & PESO & TOTAL \\
\hline Moderado & 23 & 3 & 69 \\
\hline Fraco & 38 & 2 & 76 \\
\hline Total & 19 & 1 & 19 \\
\hline Pontuação Máxima & & & 164 \\
\hline \% da Seç̧ão & & 240 \\
\hline
\end{tabular}

Fonte: Elaborado pelos autores a partir da pesquisa.

A análise da seção Aprender apresenta o resultado de desempenho médio de $68,33 \%$, com grande incidência de respostas entre moderado e fraco na instituição. No entanto, os resultados ainda são aceitáveis, segundo Bukowitz e Williams (2002). Porém, os gestores dos Cursos Tecnológicos devem observar os resultados dessa seção, principalmente porque a Gestão Acadêmica é desenvolvida na Universidade por mandatos de três anos, e o sistema eleitoral utilizado é de voto universal. Dessa forma, a probabilidade de um coordenador não se reeleger para um segundo mandato é real. Nesse cenário, caso o processo de memória organizacional não esteja claramente definido, poderá comprometer significativamente a nova gestão, com o risco de se perder todo o conhecimento e a memória desenvolvidos na gestão anterior.

$\mathrm{Na}$ seção Contribuir predomina o compartilhamento do conhecimento. $\mathrm{Na}$ coordenação dos cursos de Tecnologia em Gestão, as questões mais delicadas, neste item, estão relacionadas às barreiras impostas ao compartilhamento do conhecimento e à remoção das barreiras existentes na organização. Outro ponto importante identificado na pesquisa está no foco com que os indivíduos desempenham suas atividades em consonância com a missão da organização - esse foi um item com baixa incidência. De acordo com os resultados, percebe-se que os indivíduos desempenham as atividades relacionadas à Gestão do Conhecimento de forma instintiva, sem relacioná-las, em alguns aspectos, com a missão da organização. Outro ponto destacado, devido à baixa incidência, está no reconhecimento público na instituição do compartilhamento do conhecimento; ou seja, cada indivíduo desenvolve suas atividades sem o devido compartilhamento do conhecimento. Esses pontos foram destacados, pois obtiveram maior incidência de respostas como um ponto fraco, ou seja, são os pontos em que os respondentes menos identificaram na Gestão. 
Tabela 4 - Resumo da pontuação da seção Contribuir

\begin{tabular}{|c|c|c|c|}
\hline \multicolumn{4}{|c|}{ SEÇÃO: CONTRIBUIR } \\
\hline NÚMERO DE OCORRÊNCIAS & RESULTADO & PESO & TOTAL \\
\hline Forte & 17 & 3 & 51 \\
\hline Moderado & 42 & 2 & 84 \\
\hline Fraco & 21 & 1 & 21 \\
\hline \multicolumn{3}{|l|}{ Total } & 156 \\
\hline \multicolumn{3}{|l|}{ Pontuação Máxima } & 240 \\
\hline \multicolumn{3}{|l|}{ \% da Secção } & 65,00 \\
\hline
\end{tabular}

Fonte: Elaborado pelos autores a partir da pesquisa.

A seção Contribuir tem por objetivo identificar como ocorre o compartiIhamento do conhecimento na gestão dos cursos pesquisados. Na pesquisa foi observado uma incidência considerável de respostas fraco, ou seja, não é perceptível aos respondentes que o compartilhamento ocorra de forma consistente e institucionalizada no departamento. Essa afirmação é observada na tabela 4, pelo percentual de $65 \%$ da pontuação máxima possível.

A seção Avaliação, segundo Bukowitz e Williams (2002) está relacionada com a mensuração do conhecimento. A dificuldade de mensuração é facilmente perceptível por meio da pesquisa, visto que das 20 questões dessa seção, 5 questões obtiveram desempenho médio de 41,67\%, 6 questões com desempenho médio de $50 \%$ e 4 questões com desempenho de $58,33 \%$. As questões com desempenho médio inferior ou igual a $50 \%$ representam somadas a $55 \%$ de todas as questões da seção. Esse baixo desempenho da seção está relacionado, de acordo com os respondentes, às fragilidades na gestão acadêmica com relação a publicizar os resultados das experiências nos meios internos e externos, bem como a medição da qualidade das ações e da própria gestão do conhecimento, assim como está relacionado a uma considerável falha no mapeamento dos fluxos das atividades da gestão do conhecimento na gestão dos cursos de Tecnologia em Gestão. Esses resultados corroboram com o apresentado por Souza (2006), o qual aponta que entre as questões mais relevantes da Gestão do Conhecimento, está a mensuração do capital intelectual. 
Tabela 5 - Resumo da pontuação da seção Avaliar

\begin{tabular}{|c|c|c|c|}
\hline \multicolumn{4}{|c|}{ SEÇÃO: AVALIAR } \\
\hline NÚMERO DE OCORRÊNCIAS & RESULTADO & PESO & TOTAL \\
\hline Forte & 7 & 3 & 21 \\
\hline Moderado & 38 & 2 & 76 \\
\hline Fraco & 35 & 1 & 35 \\
\hline Total & & & 132 \\
\hline Pontuação Máxima & & & 240 \\
\hline \% da Secção & & & 55,00 \\
\hline
\end{tabular}

Fonte: Elaborado pelos autores a partir da pesquisa.

A seção Avaliar é a seção que apresenta o menor desempenho médio com $55 \%$. Do total de respostas da seção, $43,75 \%$ foram sinalizados pelos respondentes como fraco e 47,50\% como moderado. Os números indicam que a avaliação da Gestão do Conhecimento na gestão dos cursos tecnológicos precisa ser revista e que as ações em termos de gestão do conhecimento não são devidamente avaliadas e discutidas com os indivíduos envolvidos na gestão. Esses dados são preocupantes para a gestão, principalmente, em se tratando de uma instituição de ensino superior e, mais especificamente, pelos dados serem relativos à coordenação de cursos de Tecnologia em Gestão, cuja característica desta modalidade de cursos de graduação é a formação de profissionais orientados para o mercado e no atendimento de demandas específicas de mercados.

A seção Construir e Manter apresenta como resultados um desempenho individual em cada questão muito semelhante, sem muitas variações unitárias, apenas com significativo destaque, negativo, para as questões referentes à Tecnologia de Informação, cujos sistemas de informações, de acordo com os respondentes, seriam insuficientes para a realização das atividades do departamento.

Como pontos fortes, ou seja, aqueles que obtiveram maior incidência nas respostas como item que fortemente é percebido pelos indivíduos na organização, aparecem questões de disposição para aceitar ideias de outros setores ou indivíduos. Esses pontos foram avaliados de acordo com a necessidade de novos métodos de trabalho, os quais devem ser incorporados ao processo para garantir o empenho em manter pessoas com conhecimentos e capacidade indispensáveis à missão institucional. 
Tabela 6 - Resumo da pontuação da seção Construir e Manter

\begin{tabular}{l|c|c|c}
\hline \multicolumn{5}{c}{ SEÇÃO: CONSTRUIR E MANTER } \\
\hline NÚMERO DE OCORRÊNCIAS & RESULTADO & PESO & TOTAL \\
\hline Forte & 19 & 3 & 57 \\
\hline Moderado & 44 & 2 & 88 \\
\hline Fraco & 17 & 1 & 17 \\
\hline Total & & & 162 \\
\hline Pontuação Máxima & & 240 \\
\hline$\%$ da Secção & & 67,50 \\
\hline
\end{tabular}

Fonte: Elaborado pelos autores a partir da pesquisa.

A análise da pontuação da seção Construir e Manter revela que uma significativa parcela das respostas para esta seção encontra-se de forma moderada na coordenação dos cursos de Tecnologia em Gestão, obtendo um percentual de 67,50\% do desempenho máximo possível.

Os resultados da seção Descartar apresentam variação considerável, contendo questões com desempenho individual máximo e questões com desempenho baixo, oscilando entre $33,33 \%$ e $100 \%$. Neste ponto, é possível verificar como a maior fragilidade o desempenho (fraco) no intercambio de profissionais com outras instituições. E, com relação aos pontos positivos, pode ser apontada a forma humana e respeitosa como ocorrem os desligamentos de pessoal ou o encerramento de negócios e projetos.

Tabela 7 - Resumo da pontuação da seção Descartar

\begin{tabular}{l|c|c|c}
\hline \multicolumn{5}{c}{ SEÇÃO: DESCARTAR } \\
\hline NÚMERO DE OCORRÊNCIAS & RESULTADO & PESO & TOTAL \\
\hline Forte & 19 & 3 & 57 \\
\hline Moderado & 37 & 2 & 74 \\
\hline Fraco & 24 & 1 & 24 \\
\hline Total & & & 155 \\
\hline Pontuação Máxima & & 240 \\
\hline
\end{tabular}

Fonte: Elaborado pelos autores a partir da pesquisa.

O desempenho geral da seção Descartar obteve pontuação de $64,58 \%$ da pontuação máxima possível. As respostas para as questões encontram-se distribuídas com ênfase para a alternativa moderada e, na sequência, observa-se uma incidência em respostas da alternativa fraco. De acordo com os dados, é possível identificar que o descarte dos conhecimentos desnecessários ocorre na coordenação 
dos cursos. Porém, é necessário estabelecer processos claros para identificar quais os conhecimentos não são mais necessários e quanto tempo o conhecimento deve ser mantido nos repositórios.

Abaixo, o resumo geral das seções, apresentando o Diagnóstico da Gestão do Conhecimento (DGC) a partir do modelo proposto por Bukowitz e Willians (2002):

Tabela 8 - Resumo da pontuação geral das seções

\begin{tabular}{c|l|c|c|c}
\hline \multicolumn{2}{c|}{ SECÇÃO } & PONTUAÇÃO & $\begin{array}{c}\text { PESO DA } \\
\text { SECÇÃO }\end{array}$ & MEDIDA DE DESEMPENHO \\
\hline \multirow{4}{*}{ Processo Tático } & Obter & 161 & 67,08 & \\
\cline { 2 - 5 } & Utilizar & 172 & 71,67 & Melhor Desempenho \\
\cline { 2 - 5 } & Aprender & 164 & 68,33 & \\
\cline { 2 - 5 } & Contribuir & 156 & 65,00 & Pior Desempenho \\
\hline \multirow{2}{*}{$\begin{array}{c}\text { Processo } \\
\text { Estratégico }\end{array}$} & Avaliar & 132 & 55,00 & Pior Desempenho \\
\cline { 2 - 5 } & Construir e manter & 162 & 67,50 & Melhor Desempenho \\
\cline { 2 - 5 } & Descartar & 155 & 64,58 & $\mathbf{1 1 0 2}$ \\
\hline \multicolumn{3}{c}{ Total } \\
\hline \multicolumn{4}{c}{ Pontuação Máxima da Seção } \\
\hline
\end{tabular}

Fonte: Elaborado pelos autores a partir da pesquisa.

O Modelo de Diagnóstico de Gestão do Conhecimento proposto por Bukowitz e Williams (2002) aplicado à coordenação dos Cursos de Tecnologia em Gestão de uma Universidade do sul do estado de Santa Catarina, aponta para um aproveitamento da Gestão do Conhecimento de $65,60 \%$ do total da pontuação máxima possível. Pontuação esta que, segundo apresentado pelas autoras, é considerado bom, visto que os índices sugeridos aferem como uma boa gestão do conhecimento índices próximos de $55 \%$.

O Modelo de Diagnóstico é estruturado em dois processos macro (processos táticos e processos estratégicos) e cada processo engloba as seções. Nos processos táticos que compreendem os processos de Obter, Utilizar, Aprender e Contribuir, a seção que apresentou menor desempenho foi a Contribuir, com $65 \%$ e o melhor desempenho está na seção Utilizar, com $71,67 \%$. Tais índices, de acordo com o modelo, são considerados bons em termos de avaliação da Gestão do Conhecimento. Nos processos estratégicos que compreendem as seções Avaliar, Construir/Manter e Descartar, observa-se o melhor desempenho na seção Construir/Manter, com $67,50 \%$ da pontuação máxima para a seção. 0 pior desempenho é da seção Avaliar, com 55\%. 
O Modelo de Diagnostico de Gestão do Conhecimento (DGC) de Bukowitz e Williams (2002) considera que uma boa gestão do conhecimento apresenta valores médios por seção entre $30 \%$ e $70 \%$.

\section{CONSIDERAÇÕES FINAIS}

O modelo de Gestão do Conhecimento é dividido em sete seções, segundo Bukowitz e Williams (2002). O desempenho médio das seções para considerar que a empresa trabalha de acordo com a teoria da Gestão do Conhecimento deve obter índices que variam de 30 a 70\% nas seções e de 55\% no geral. Dessa forma, podese inferir que a Gestão do Conhecimento aplicada na gestão acadêmica dos Cursos de Tecnologia em Gestão, embora não existam programas claramente definidos para a Gestão do Conhecimento, está executando os processos satisfatoriamente, visto que o desempenho médio geral atingiu $65,60 \%$.

No entanto, percebe-se que há um longo caminho a ser percorrido para melhorar os processos da Gestão do Conhecimento nos cursos, uma vez que os menores percentuais de desempenho se encontram nos processos estratégicos; o pior desempenho está no processo de Avaliar, com 55\%, seguido do processo Descartar. Diante desses resultados, pode-se concluir que a gestão acadêmica apresenta fragilidades na Gestão do Conhecimento nos processos estratégicos, principalmente, na avaliação e mensuração dos resultados do conhecimento e no descarte das informações desnecessárias. Esses fatores podem ser explicados, conforme entendimento de Hardy e Fachin (2000), uma vez que a gestão acadêmica é desenvolvida por professores, que, devido à elevada carga de trabalho, podem interferir, substancialmente, no desenvolvimento dos processos de Gestão do Conhecimento. Outro fator que interfere está relacionado às mudanças na gestão acadêmica, uma vez que os coordenadores são eleitos ou indicados pela reitoria e os mandatos têm duração de três anos. Dessa forma, nos momentos de transição de coordenação, muitas informações e processos podem ser perdidos pela falta de processos estruturados de compartilhamento do conhecimento.

$\mathrm{Na}$ análise da Gestão do Conhecimento foi possível constatar que existe grande quantidade de informações e conhecimentos gerados, bem como utilizados, os quais contribuem para o aprendizado nos processos táticos. Não obstante, a grande fragilidade está nos processos estratégicos, na avaliação e no descarte. Diante disso, sugerem-se novos estudos para buscar alternativas que possam apresentar 
subsídios para melhor estruturar a Gestão do Conhecimento na coordenação dos Cursos de Tecnologia em Gestão e ampliar os estudos na IES.

\section{REFERÊNCIAS}

ALMEIDA, Denise Ribeiro de. Processo gestor do ensino superior federal: uma análise à luz da experiência UFBA. Contabilidade, Gestão e Governança - Brasília. v. 17, n. 1, p. 4-17, jan./abr. 2014.

BRASIL. PRESIDÊNCIA DA REPÚBLICA. Lei n. 9.394/96. Lei de Diretrizes e Bases da Educação Nacional. MEC, Brasília, 20 de dezembro de 1996.

BRITO, Lydia Maria Pinto; BOLSON, Saionara Brano. Gestão do conhecimento: estudo em uma instituição privada de ensino superior. Revista Raunp, v. 6, n. 2, p. 77-87, abr./set. 2014. ISSN 1984-4204.

BUKOWITZ, W. R.; WILLIAMS, R. L. Manual de gestão do conhecimento: ferramentas e técnicas que criam valor para a empresa. Porto Alegre: Bookman, 2002. Tradução Carlos Alberto Silveira Netto Soares.

COSTA, I.; et al. Diagnóstico de gestão do conhecimento como mecanismo para criação de valor: um estudo exploratório no Sebrae-PB. Revista Gestão Industrial, Ponta Grossa, v. 5, p. 80-98, abr./jun. 2009.

DIHL, Winicyus; HOLANDA, Lucyanno Moreira Cardoso de; FRANCISCO, Antonio Carlos de. A utilização do método de diagnóstico para a gestão do conhecimento: estudo de caso numa empresa incubada (ALFA). Revista Eletrônica FAFIT/FACIC. v. 1, n. 1, jan./jun. 2010, p. 30-43.

FACHIN, Odília. Fundamentos de metodologia. 4. ed. São Paulo: Saraiva, 2003.

GIL, Antônio Carlos. Métodos e técnicas de pesquisa social. 5. ed. São Paulo: Atlas, 2006.

HARDY, C.; FACHIN, R. Gestão estratégica na universidade brasileira: teoria e casos. 2. ed. Porto Alegre: UFRGS, 2000.

JACOMETTI, Márcio. Cultura organizacional e interesses na definição de estratégias: o caso de uma Instituição de Ensino Superior. Organizações em contexto, São Bernardo do Campo, ISSNe 1982-8756, v. 8, n. 15, jan.-jun. 2012 
MARRA, A. V.; MELO, M. C. O. L. A prática social de gerentes universitários em uma instituição pública. Revista de Administração Contemporânea [online]. 2005, v. 9, n. 3, p. 9-31, 2005. Disponível em: <http://www.scielo.br/scielo.php?pid=S141565552005000300002\&script=sci_arttext>. Acesso em: 28 dez. 2012.

MELO, Marlene Catatina de Oliveira Lopes; LOPES, Ana Lúcia Magri; RIBEIRO, João Marcelo. $O$ cotidiano de gestores entre as estruturas acadêmica e administrativa de uma instituição de ensino superior federal de Minas Gerais. Organizações em contexto, São Bernardo do Campo, ISSNe 1982-8756, v. 9, n. 17, jan.-jun. 2013

MIRANDA, A. R. A. Um estudo sobre a dinâmica identitária de professoras gerentes de uma universidade pública. 2010. 162 f. Dissertação (Mestrado em Administração) - Programa de Pós-Graduação em Administração. Universidade Federal de Lavras. Lavras, Minas Gerais, 2010.

NONAKA, Ikujiro; TAKEUCHI, Hirotaka. Criação de conhecimento na empresa. Rio de Janeiro: Campus, 1997.

SAUSSOIS, J. M. Coordenador, coopérer, adhérer - Les enjeux du management. Sciences Humaines, n. 20, mars./avr. 1998. (Hours Serie).

SILVEIRA, Mônica Maria Rebelo Velloso da; NETO, Ivan Rocha. Gestão do conhecimento e a oralidade na CAPES: implicações à inteligência coletiva. Perspectivas em Gestão \& Conhecimento, João Pessoa, v. 3, número especial, p. 148-162, out. 2013.

SOUZA, Daniela Borges Lima de. Gestão do conhecimento nas organizações: desafios e oportunidades. Estação Científica, Juiz de Fora, n. 3, out. 2006.

TARDIF, M. Saberes profissionais dos professores e conhecimentos universitários. Revista Brasileira de Educação, n. 13, p. 5-24, jan./fev./mar./abr. 2000.

TERRA, J. C. C. Gestão do conhecimento: o grande desafio empresarial. Rio de Janeiro: Negócio, 2000. 